\title{
Lateral Translation of the Patella in MPFC Reconstruction: A Biomechanical Study of Three Approaches
}

\author{
William M. Cregar, MD ${ }^{1}$ Hailey P. Huddleston, BS ${ }^{1} \quad$ Elizabeth F. Shewman, MS ${ }^{1}$ Brian J. Cole, MD, MBA ${ }^{1}$ \\ Adam B. Yanke, MD, $\mathrm{PhD}^{1}$ \\ ${ }^{1}$ Division of Orthopedic Sports Medicine, Rush University Medical \\ Center, Chicago, Illinois \\ J Knee Surg 2023;36:622-630. \\ Address for correspondence Adam B. Yanke, MD, PhD, Division of \\ Orthopedic Sports Medicine, Midwest Orthopedics at Rush University \\ Medical Center, 1611 West Harrison Street, Suite 300, Chicago, IL \\ 60612 (e-mail: adam.yanke@rushortho.com).
}

\begin{abstract}
Keywords

- knee

- medial patellofemoral complex

- patellar instability

The purpose of this study was to investigate whether differences exist in preventing lateral patellar translation between three distinct medial patellofemoral complex (MPFC) reconstruction procedures at varying knee flexion angles. Six cadaveric knee specimens were dissected, potted, and placed in a customized jig for testing. Lateral patellar displacement was measured at intervals between 0 and 90 degrees of knee flexion using a tensile testing machine with a $20 \mathrm{~N}$ lateral force applied to the patella. Each specimen was tested with the MPFC intact, sectioned, and after each of the three reconstruction techniques: MPFL, hybrid, and medial quadriceps-tendon femoral (MQTFL) reconstructions. There was significantly increased lateral patellar displacement following MPFC sectioning when compared with the intact state in early degrees of flexion $(10-30$ degrees $)(p<0.05)$. All three reconstruction groups restored patella stability and reduced lateral patellar displacement following sectioning from 0 to 30 degrees of flexion $(p<0.05)$. When compared with the intact group, all three reconstruction groups demonstrated reduced patella translation at full knee extension, while the MPFL and hybrid reconstruction groups additionally demonstrated significant reduction in patella translation at 10 degrees of flexion $(p<0.05)$. No significant differences were observed between the three reconstruction groups. This biomechanical study demonstrates the efficacy of three MPFC reconstruction techniques in patella stabilization following sectioning. Our results suggest that MPFL reconstruction may provide the most robust patella stabilization, whereas MQTFL reconstruction may be the most forgiving construct. This study suggests that MQTFL and hybrid reconstructions provide adequate resistance to lateral translation and may be used as an alternative to MPFL reconstruction.
\end{abstract}

Patellar dislocation is a common knee disorder affecting predominantly adolescents and can lead to recurrent subluxation or dislocation, chronic pain, and functional disability. $^{1-3}$ Lateral patellar dislocation leads to significant disruption of the medial soft tissue stabilizers, with the medial patellofemoral ligament (MPFL) being the most important, historically providing up to $60 \%$ of static medial restraint against lateral patellar movement. ${ }^{4}$ More recently, Tanaka et al demonstrated that while the MPFL is an important medial patella stabilizer, it is only one of several other received

June 30, 2021

accepted after revision

November 16, 2021

article published online

February 10, 2022 (c) 2022. Thieme. All rights reserved.

Thieme Medical Publishers, Inc., 333 Seventh Avenue, 18th Floor, New York, NY 10001, USA
DOI https://doi.org/ 10.1055/s-0041-1741549. ISSN 1538-8506. 
anatomical medial retinacular structures that collectively comprise the medial patellofemoral complex (MPFC). ${ }^{3}$ This complex includes the proximal medial patellar restraints (PMPR) that are composed of the MPFL and medial quadriceps-tendon femoral ligament (MQTFL) and the distal medial patellar restraints that are composed of the medial patellotibial ligament (MPTL) and medial patellomeniscal ligament (MPML). ${ }^{3}$

Surgically addressing recurrent patella instability has classically focused on reconstruction of the MPFL, which has widely become the standard of care either in isolation or concomitantly with other patellar realignment procedures. ${ }^{4-9}$ Complications following MPFL reconstruction include patellar fracture, articular surface penetration, and physeal injury in immature pediatric patients. ${ }^{10,11}$ In efforts to avoid these, other surgical techniques have been described. These include physeal sparing techniques as well as techniques that avoid or limit osseous patella fixation such as MQTFL reconstruction described by Fulkerson and Edgar and PMPR, or "hybrid," reconstruction described by Dr. Farr. ${ }^{12}$ Previous biomechanics testing has demonstrated the MPFL's role as the primary lateral patellar restraint, while the MQTFL appears to provide its greatest action against lateral patellar movement when the knee is in full extension. ${ }^{13}$ While these alternative MPFC reconstructions have anatomical support and the theoretical potential to reduce complications, it is unknown whether differences exist in lateral patellar translation between reconstruction techniques and thus their effectiveness in adequately stabilizing the patella.

The purpose of this study was to investigate whether a difference in lateral patellar translation exists between three distinct medial patellar stabilizing surgical procedures at varying knee flexion angles. We hypothesized that all three MPFC reconstruction techniques will decrease lateral patellar translation, when compared with specimens with sectioned native medial soft tissue patellar restraints. We also hypothesized that the three MPFC reconstruction techniques will differ in ability to stabilize the patella with higher amounts of soft tissue stabilization providing inferior results and thus higher amounts of lateral patellar translation when compared with techniques with stouter osseous patella fixation.

\section{Methods}

\section{Specimen Preparation}

Six nonpaired, fresh-frozen human cadaveric knees (Allosource, Centennial, CO), without previous injury, surgery, or a history of knee arthritis, were utilized for this study. Inclusion criteria for the selected knee specimens included age $<65$, body mass index $<35$, those without previous injury, surgery or a history of knee osteoarthritis, cancer that metastasized to bone, and history of being bedridden. The mean age was $58.3 \pm 4.63$ years, there was a ratio of $4: 2$ females to males, and a ratio of $4: 2$ left to right knees. Specimens were stored in a -20 degrees freezer and thawed overnight prior to initiation of the study.

For test preparation, the femur and tibia and fibula were cut $\sim 20 \mathrm{~cm}$ from the joint line (proximal for femur, distal for the tibia and fibula). Skin, subcutaneous tissue, fascia, and muscle over the proximal femur and distal tibia and fibula were removed circumferentially to expose the underlying bone while leaving the proximal portion of the interosseous membrane intact on the tibia and fibula. The tibia and fibula were potted axially $\sim 11 \mathrm{~cm}$ distal to the tibial tubercle in a cylindrical mold using polymethyl methacrylate (Isocryl; Lang Dental, Wheeling, IL). The femur was potted in a similar manner, $\sim 15 \mathrm{~cm}$ proximal to the joint line. After potting, the midpole of the patella was identified and a skin incision was made at the lateral aspect of the patella with dissection carried down to bone, ensuring to stay extra-articular. A small pilot hole was drilled into the midpoint of the patella with subsequent insertion of a $6.3 \mathrm{~mm}$ diameter eye screw, again ensuring to remain extra-articular. Each specimen underwent arthroscopic evaluation to confirm the presence of a continuous, intact MPFC as well as no patellofemoral abnormalities or severe arthritic changes throughout the knee. Extra-articular patellar screw placement was also confirmed arthroscopically. The quadriceps were then separated into three components: vastus lateralis (VL), vastus medialis obliquus (VMO), and combined rectus femoris/vastus intermedius (RF/VI) muscle bellies. Running Krackow sutures were placed into the tendinous portion of each of the three quadricep components and tied at their free ends to create a loop such that a weighted load could be placed on the extensor mechanism during testing.

\section{Experimental Design}

This study was exempt from institutional institutional review board approval due to the use of de-identified cadaveric specimens. Each knee specimen underwent five rounds of lateral patella translation testing coordinating with five different states: intact, sectioned (native MPFL and MQTFL sacrifice), and following each of the three reconstruction techniques (MPFL reconstruction, MQTFL reconstruction, and Hybrid/PMPR reconstruction) in a randomized order (-Fig. 1). Unique frozen semitendinosis allografts thawed to room temperature were used in all reconstruction techniques

\section{Native MPFL/MQTFL Sectioning}

An incision was made from the superomedial border of the patella extending distally to the midpole of the patella along its medial aspect. Layers 1 and 2 were sharply incised with dissection proceeding down to capsule (ensuring sacrifice of both the native MPFL and MQTFL). Staying extracapsular, a plane was bluntly dissected medially between layers 2 and 3 toward the MPFL femoral insertion point. The VMO was visualized proximally within the wound to ensure all proximal medial soft tissue restraints were sacrificed. Grossly increased manual lateral patellar translation was used to secondarily confirm adequate sectioning.

\section{Medial Patellofemoral Ligament (MPFL) Reconstruction Technique}

The incision placed to section medial patellar restraints as described above was used for patella fixation in MPFL 


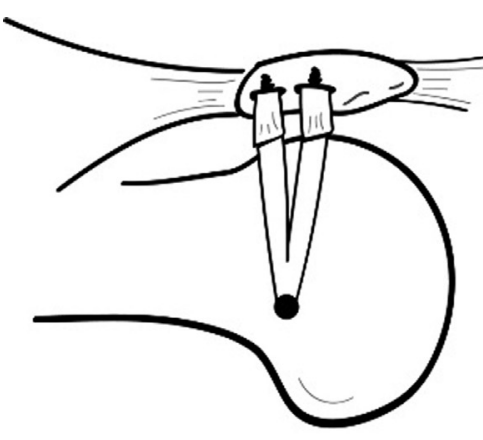

MPFL Reconstruction

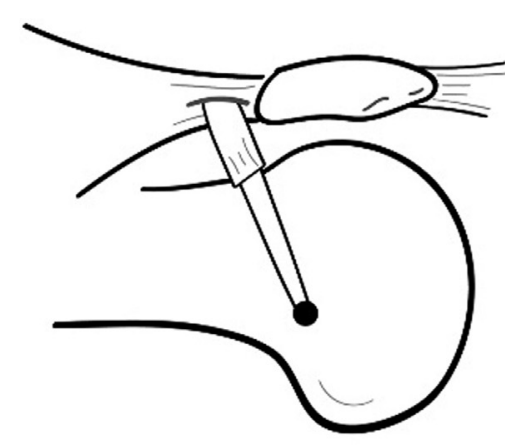

MQTFL Reconstruction

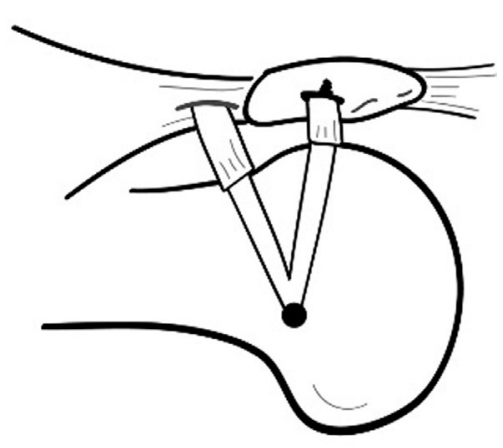

Hybrid Reconstruction

Fig. 1 Pictorial representation of each of the three MPFC reconstruction techniques using semi-tendinosis allograft. MPFL, medial patellofemoral ligament; MQTFL, medial quadriceps-tendon femoral ligament.

reconstruction. The superomedial aspect of the patella was identified with sharp dissection down to bone. A small bony defect was created within the medial patella using a rongeur to prepare a crevice for suture anchor placement. A $3.0 \mathrm{~mm}$ polyether ether ketone (PEEK) suture anchor was then placed within the superomedial aspect of the patella. A $3.0 \mathrm{~mm}$ PEEK suture anchor was then placed at the midpole of the patella in a similar manner. A generous incision was then placed over the medial aspect of the knee centered over the medial epicondyle. Dissection was carried down through subcutaneous tissue until a thickening was felt running horizontally toward the saddle point between the adductor tubercle and medial epicondyle, which represented the femoral insertion point of the native MPFL. The ligament was traced to its exact femoral insertion and a beath pin was placed and directed medial to lateral in a slightly proximal direction. Dissection was performed between layers 2 and 3 toward the MPFL femoral insertion point. This plane was opened to allow room for passage of the graft. Suture limbs from each patella suture anchor were tunneled between layers 2 and 3, tensioned, and clamped around the embedded beath pin with the knee in full extension. The knee was then ranged from 0 to 90 degrees of flexion to ensure isometry of the suture limbs confirming proper femoral tunnel positioning. The beath pin was then unicortically overdrilled to a depth to allow graft fit. The semitendinosus allograft $(6-8 \mathrm{~mm}$ in diameter, Allosource, Centennial, $\mathrm{CO}$ ) was then secured into the femoral tunnel with an interference screw fixation such that the two graft ends were free for patella fixation. The graft ends were tunneled laterally between layers 2 and 3 and brought up to the medial aspect of the patella. The patella was centered within the trochlea with the knee in full extension and both graft limbs were independently secured to each patella suture anchor through knot fixation. The remaining distal ends of the graft limbs were then brought back down onto themselves and secured with nonabsorbable suture for extra fixation (-Fig. 1).

\section{Medial Quadriceps Tendon Femoral Ligament (MQTFL) Reconstruction Technique}

MQTFL reconstruction was performed as described by Fulkerson and Edgar (-Fig. 1). ${ }^{12}$ The same skin incision per- formed for medial restraint sectioning was used with slight proximal extension of the incision to identify the VMO and quadriceps tendon junction. At the superior aspect of the patella $\sim 1 \mathrm{~cm}$ lateral to the VMO-quadriceps tendon junction, a small $2 \mathrm{~cm}$ incision was made within the quadriceps tendon. Sharp dissection was carried down through the tendon ensuring to stay extracapsular. A hemostat was placed through the quadriceps incision and tunneled laterally underneath the VMO in between layers 2 and 3 to ensure enough space for graft passage. The femoral tunnel was prepared in a similar manner as described above for MPFL reconstruction. The semitendinosus allograft was secured within the femoral tunnel in a similar manner to MPFL reconstruction; however, only one graft limb was brought medially between layers 2 and 3, underneath the VMO and tunneled out of the quadriceps tendon from posterior to anterior. The patella was centered within the trochlea with the knee in extension and the free end of the graft was then brought back down medially and secured to itself with two separate suture knots. The other inferior allograft limb was left free and not included within this construct.

\section{Hybrid Reconstruction Technique-Proximal Medial Patellar Restraints (PMPR) Reconstruction}

The PMPR reconstruction, also deemed the "Hybrid Technique," was performed according to that conceptualized by Dr. Jack Farr, which has not yet been published within the literature (-Fig. 1). The femoral tunnel positioning and semitendinosus allograft fixation were the same as that described in both the MPFL and MQTFL reconstruction techniques. With the patella appropriately centered, the superior limb of the graft was tunneled between layers 2 and 3, underneath the VMO, up through the quadriceps tendon and secured back onto itself exactly how it was described in the MQTFL reconstruction technique above. The inferior limb of the graft was tunneled laterally between layers 2 and 3 and secured to the inferior patellar suture anchor through knot fixation as described above within the MPFL reconstruction technique. Similarly, for extra security the graft end was brought back onto itself and secured with suture knot fixation. 


\section{Experimental Testing}

All six knees underwent lateral patellar translation testing using the MTS tensile machine (Insight 5, MTS Inc, Eden Prairie, MS) for each of five groups: 1. intact, 2. sectioned, 3. following isolated MQTFL reconstruction, 4. following MPFL reconstruction and after 5 . hybrid/PMPR reconstruction, with the three different reconstruction groups tested in a random order. Each test group was analyzed at 0, 10, 20, 30, 45, 60, 90 degrees of knee flexion. All knees were secured into a custom machined jig such that the knee was perpendicular to the lateral patellar force vector (-Fig. 2). The lateral patella eye screw was secured to the load cell of the tensile machine by a steel cable. A total of $175 \mathrm{~N}$ load was distributed over the three quadricep components in a proximal vector to simulate in vivo quadriceps activation as described previously. ${ }^{16}$ The total quadriceps load was distributed between the VL, VM, and RF/VI with 35,25 , and $40 \%$ of the total force, respectively, using a custom pulley system. During lateral patellar translational testing, a direct lateral force was applied to the patella through the laterally placed eyelet screw at a rate of $1.0 \mathrm{~mm} / \mathrm{sec}$ until a $20 \mathrm{~N}$ load was achieved. Each knee was then tested at flexion angles between 0 and 90 degrees as described above. Load and displacement data was collected at $40 \mathrm{~Hz}$ and saved on a personal computer using the TestWorks software (Insight 5 , MTS Inc, Eden Prairie, MS). The primary outcome measure was the lateral translation of the patella between $0.1 \mathrm{~N}$ and $20 \mathrm{~N}$ and was determined at each increment.

\section{Power Analysis}

A power analysis was performed to estimate the necessary sample size. An a priori analysis was performed using $G^{*}$ power. We investigated the necessary change in lateral displacement needed between intact or medial sectioning compared with each reconstruction group to reach significance. A correlation between the variables was set at 0.9 based on pilot data and previously published results

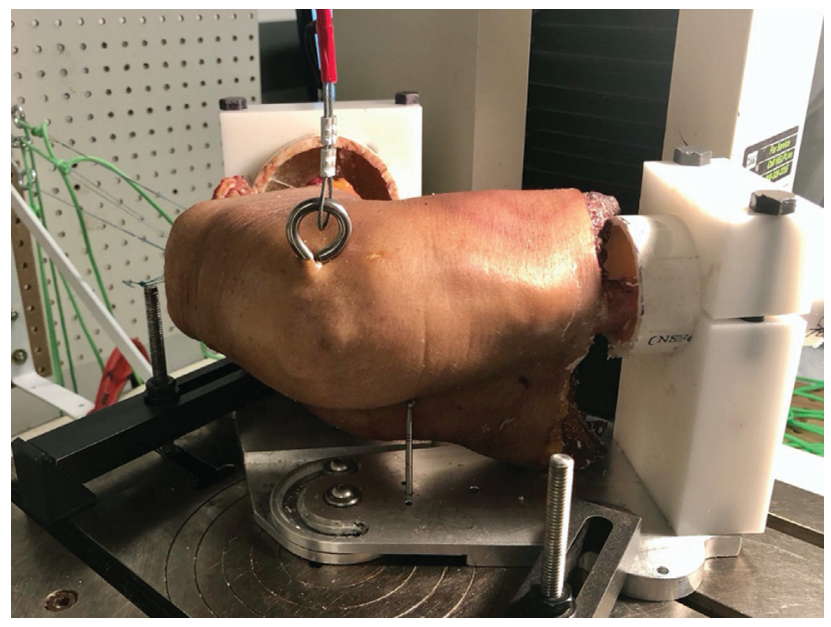

Fig. 2 Experimental testing setup. Photograph of a potted cadaver knee secured into a customized jig positioned perpendicular to the tensile testing machine that exerts its lateral patellar force vector through a steel cable connected to the lateral patellar eye screw. analyzed using a spearman correlation in STATA (v13, StataCorp, College Station, TX). ${ }^{13,14}$ With a difference in mean of $4 \mathrm{~mm}$ and a standard deviation of $5 \mathrm{~mm}$, a power of $80 \%$, and an $\alpha$ of 0.05 , a one tailed $t$-test needed a sample size of four to reach significance.

\section{Statistical Analysis}

Statistical analysis was conducted using SPSS (version 25; IBM, Armonk, NY). The displacement data was not normally distributed; therefore, all displacement data are presented as medians. To compare differences in displacement between more than two groups, a Friedman test was utilized for each tested degree of flexion $(0,10,20,30,45,60,90$ degrees $)$. When displacement was compared between two groups, a Wilcoxon rank sum test was used at each tested degree of flexion. Significance was set at $p<0.05$, whereas trending toward significance was set at $p<0.1$.

\section{Results}

All knees had an intact MPFC without evidence of osteoarthritis confirmed by arthroscopy. Median values for lateral patellar displacement of the intact MPFC, sectioned MPFC, and all three reconstruction groups are shown in - Fig. 3. The findings of one specimen were removed from the sectioned group due to incomplete MPFC sectioning. Thus, five specimens were included in the final sectioned MPFC cohort. When comparing lateral displacement of the intact MPFC to the sectioned MPFC, there was a significant increase in lateral patellar displacement in the MPFC sectioned groups at $10(p=0.040), 20(p=0.040)$, and 30 degrees $(p=0.040)$. At 0 and 45 degrees of flexion, the differences trended toward significance ( $p=0.08$ for both) ( - Fig. 4 ). There was a small but significant decrease in lateral patella translation seen at 60 degrees of knee flexion $(p=0.040)$ in the sectioned state as compared with the intact native state.

\section{Comparison to Intact State}

Lateral patellar displacement of each reconstruction group was compared with the intact MPFC state (-Fig. 5). In the MPFL reconstruction group, there was significantly decreased lateral patellar translation at $0(p=0.028)$ and 10 degrees of flexion $(p=0.046)$; however, there was only a trend toward significance at $20(p=0.075)$ and 30 degrees of flexion $(p=0.075)$. In the hybrid reconstruction group, significantly decreased lateral patellar displacement was observed at $0(p=0.028), 10(p=0.046)$, and 30 degrees of flexion $(p=0.028)$ and only trended toward significance at 20 degrees of flexion $(p=0.075)$. Finally, in the MQTFL reconstruction group, lateral patellar translation was significantly decreased at 0 degrees of flexion $(p=0.046)$ and only trended toward significance at 10 degrees of flexion $(p=0.075)$.

\section{Comparison to Sectioned State}

Lateral patellar displacement of each reconstruction group was then compared with the MPFC sectioned state (-Fig. 6 ). The lateral displacement of the MPFC sectioned state was 


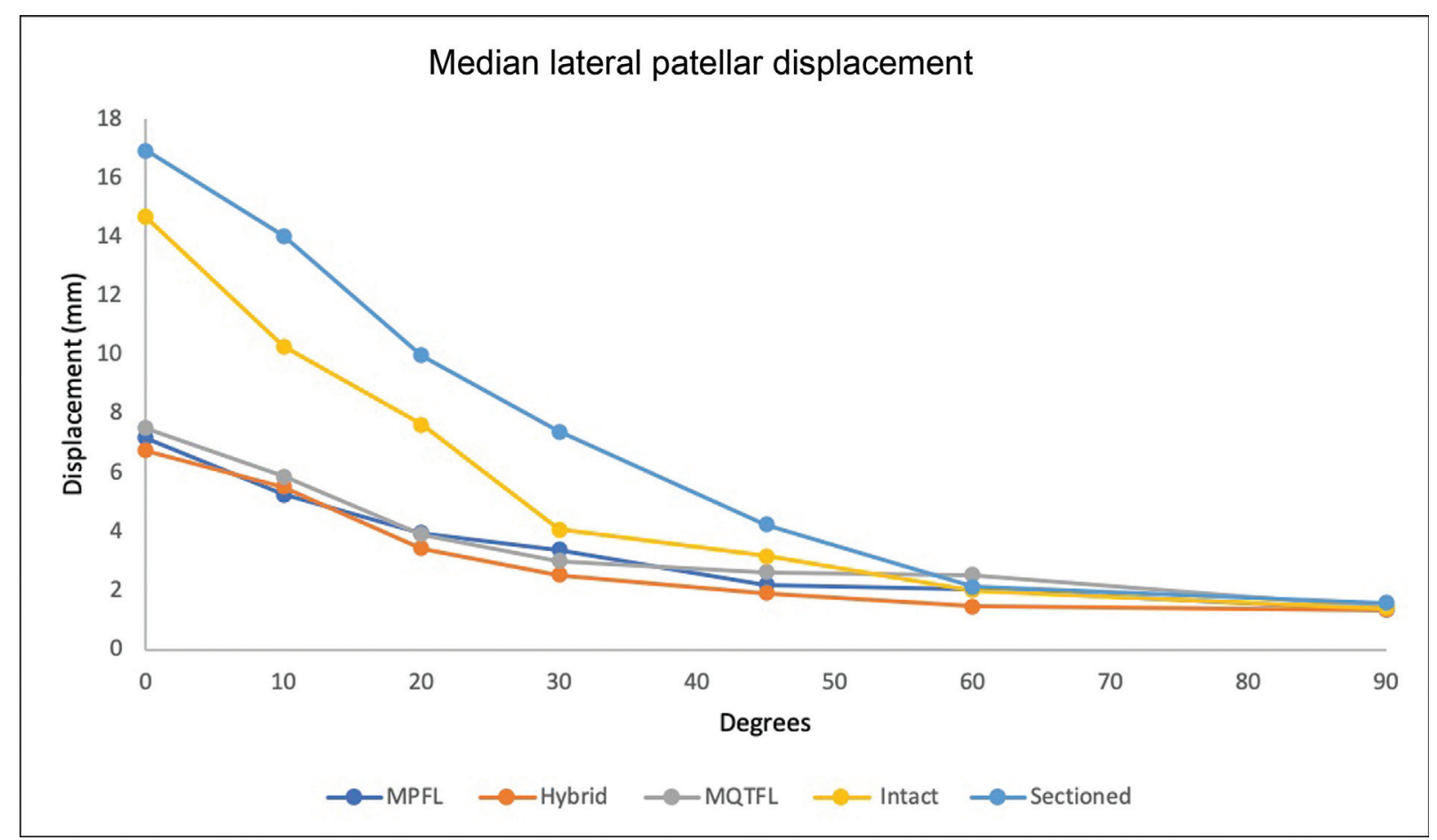

Fig. 3 Median lateral patellar displacement of the intact MPFC state, the sectioned MPFC state, and each of the three MPFC reconstruction groups (MPFL reconstruction, hybrid reconstruction, and MQTFL reconstruction). MPFC, medial patellofemoral complex; MPFL, medial patellofemoral ligament; MQTFL, medial quadriceps-tendon femoral ligament.

significantly greater than that of each reconstruction group at $0,10,20$, and 30 degrees of flexion $(p<0.05)$. When compared with the sectioned state at early degrees of knee flexion (0-30 degrees), lateral patella translation was reduced by 42,41 , and $33 \%$ following MPFL, MQTFL, and hybrid reconstructions, respectively.

\section{Comparison between Reconstruction Groups}

Lateral patellar displacement among the three reconstruction groups was compared with results shown in $\sim$ Fig. 7. Friedman analysis of the three groups found significant differences in displacement at 0 degrees of flexion $(p=0.042)$, with MPFL reconstruction demonstrating the smallest lateral patella

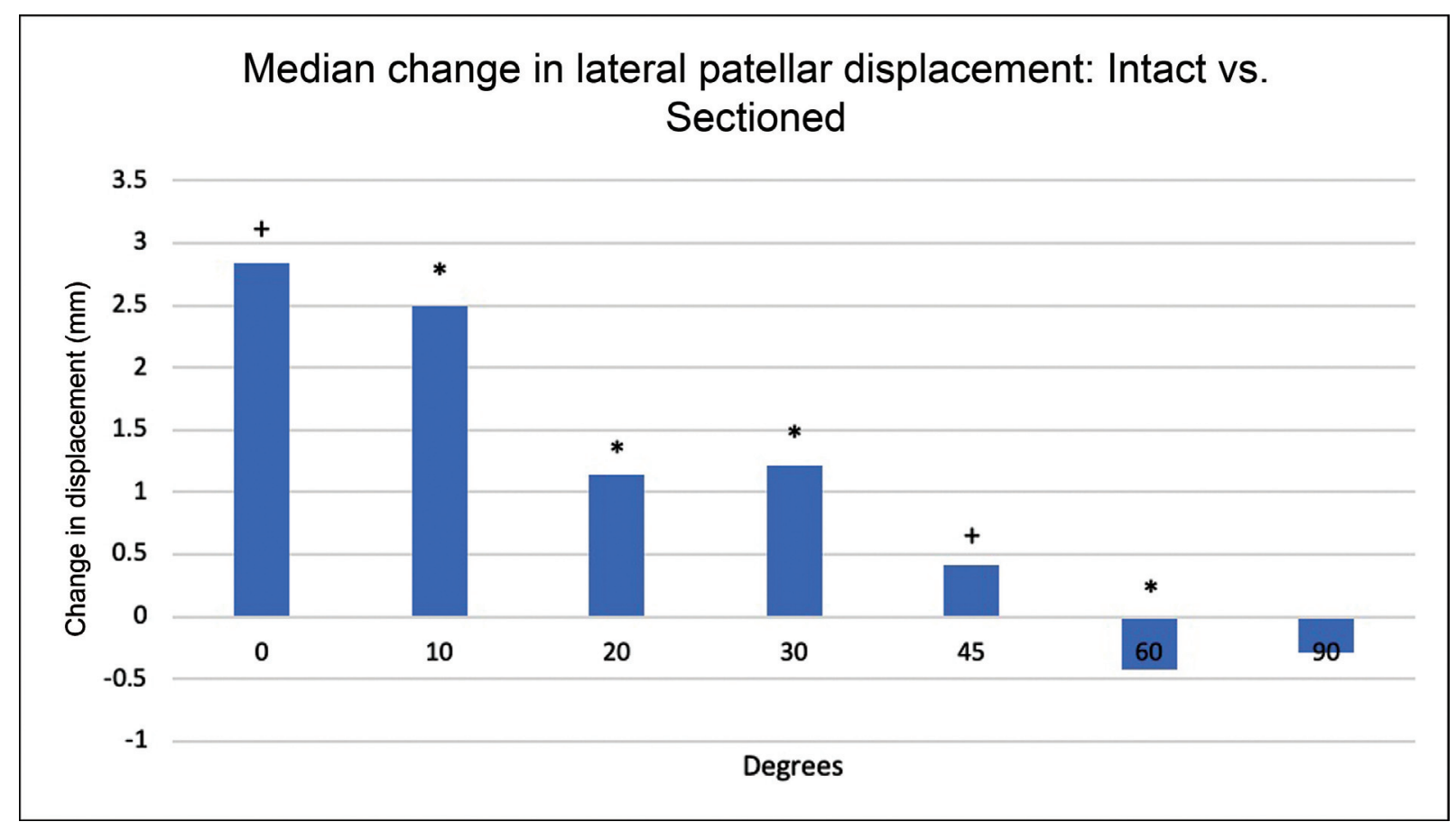

Fig. 4 Median difference in lateral patellar displacement from intact MPFC state to sectioned MPFC state. ${ }^{*} p<0.05 ;+p<0.10$; MPFC, medial patellofemoral complex. 


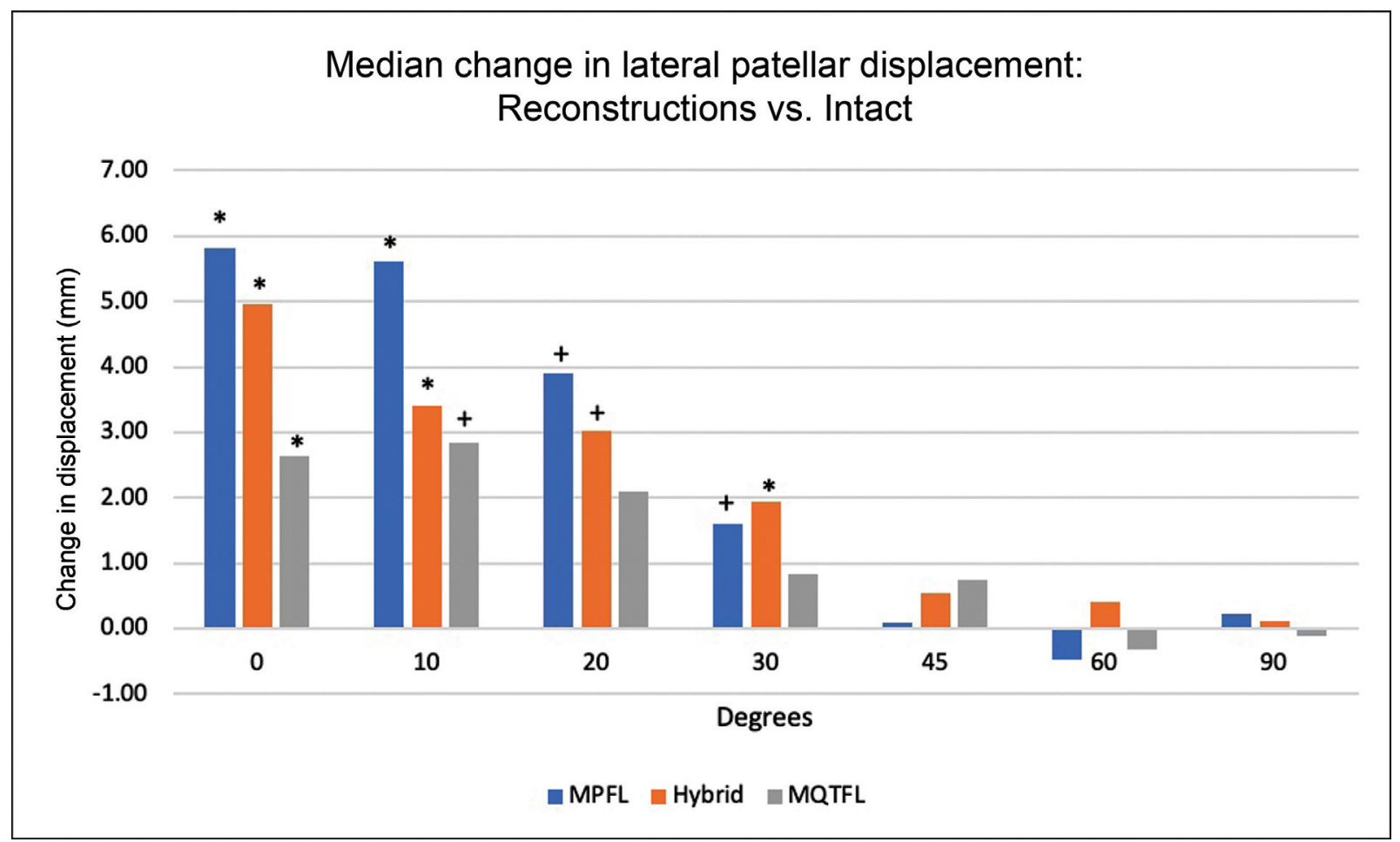

Fig. 5 Median difference in lateral patellar displacement between the intact MPFC state and each MPFC reconstruction state. ${ }^{*} p<0.05 ;+$ $p<0.10$; MPFC, medial patellofemoral complex; MPFL, medial patellofemoral ligament; MQTFL, medial quadriceps-tendon femoral ligament.

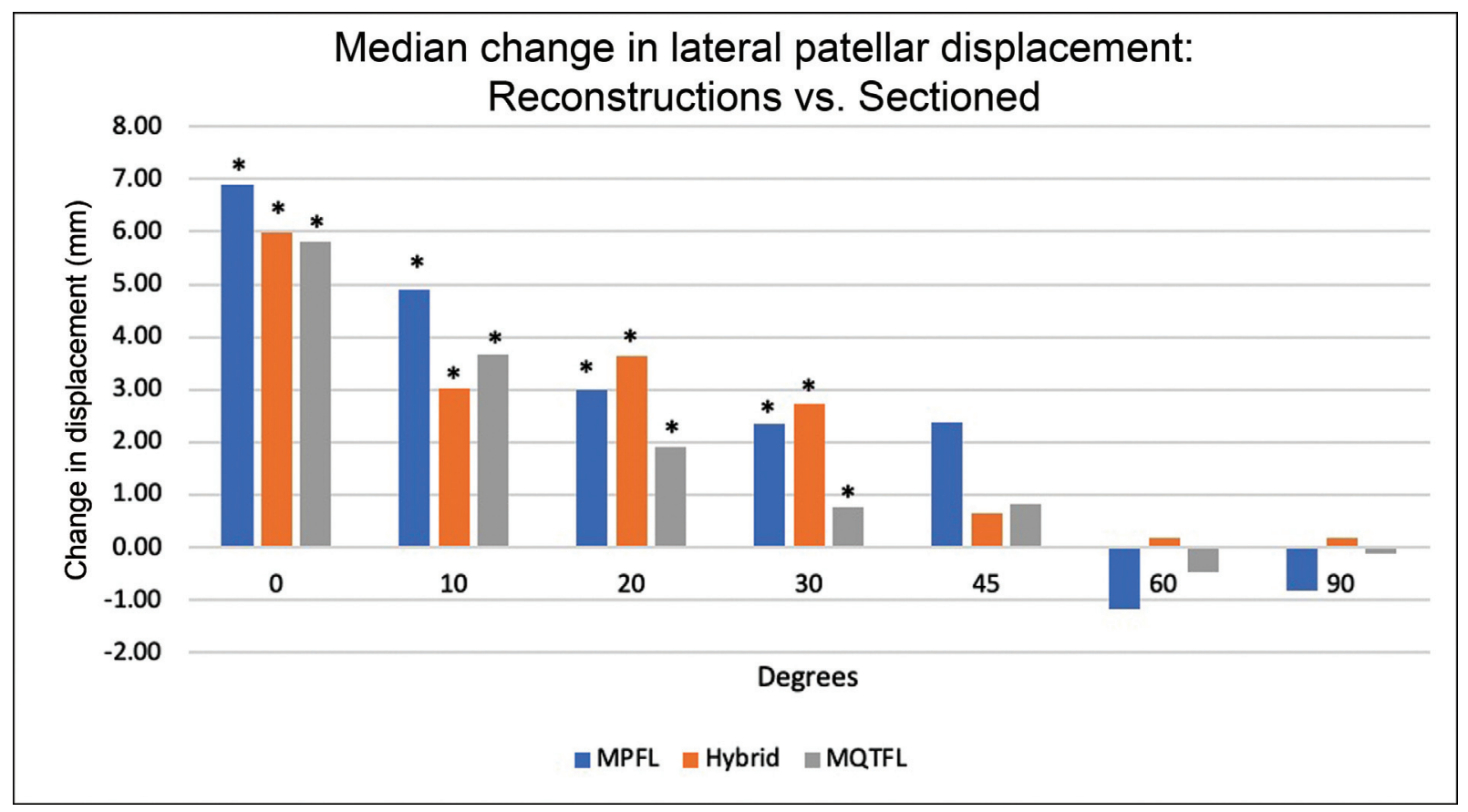

Fig. 6 Median difference in lateral patellar displacement between the sectioned MPFC state and each reconstruction group. ${ }^{*} p<0.05$; MPFC, medial patellofemoral complex; MPFL, medial patellofemoral ligament; MQTFL, medial quadriceps-tendon femoral ligament.

translation and MQTFL reconstruction demonstrating the largest. Post-hoc pairwise comparisons found that differences between the MPFL reconstruction and MQTFL reconstruction only trended toward significance $(p=0.063)$. No significant differences in lateral patellar displacement were observed between the MPFL, hybrid, and MQTFL reconstruction groups at 10 to 90 degrees of knee flexion.

\section{Discussion}

This study evaluated the efficacy of restoring anatomic medial patellar restraints and preventing lateral patella translation following MPFC sectioning and subsequent MPFL, MQTFL, and hybrid reconstructions. Our study demonstrated that MPFC sectioning led to increased lateral 


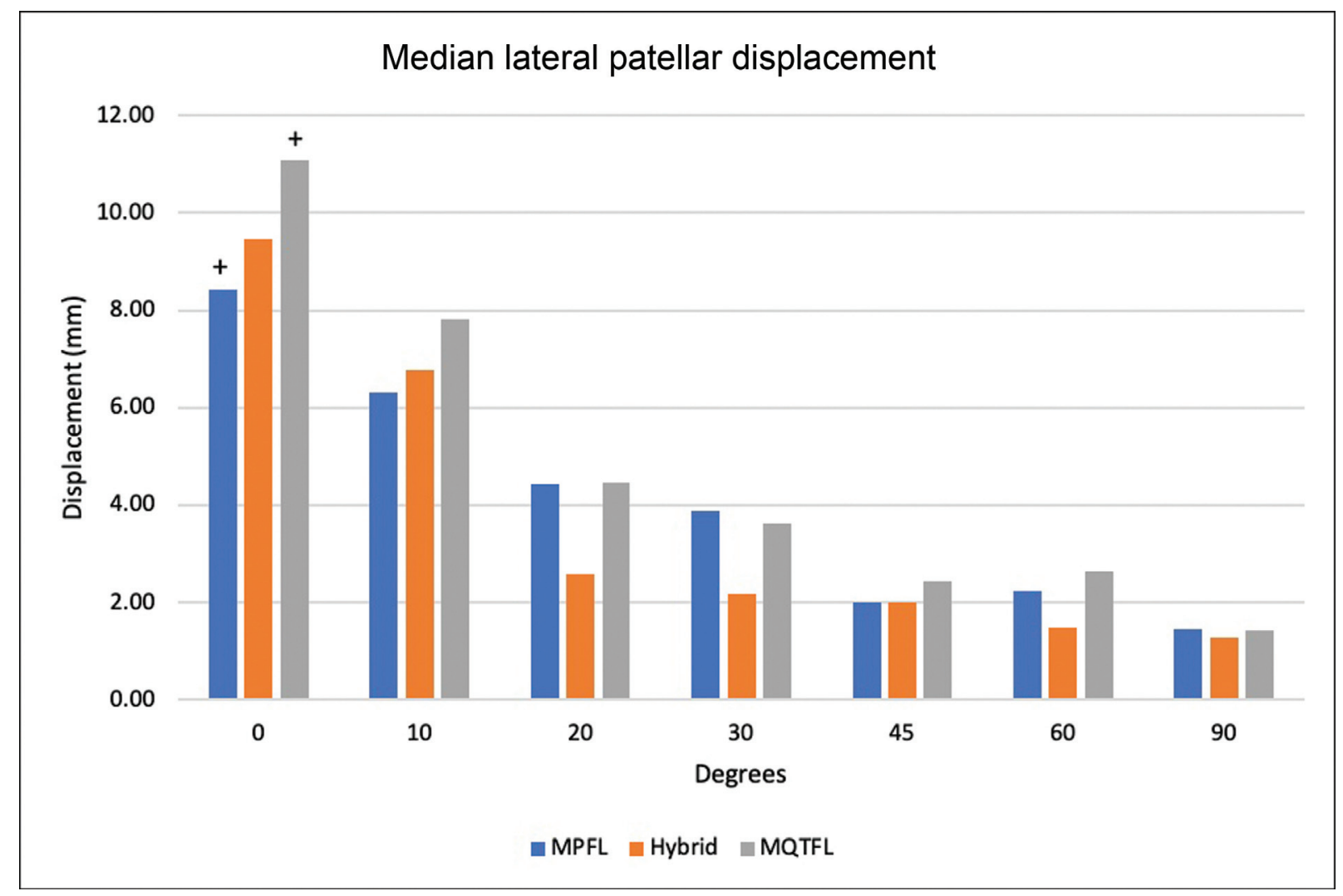

Fig. 7 Median lateral patellar displacement between each MPFC reconstruction state. ${ }^{*} p<0.05 ;+p<0.10$; MPFC, medial patellofemoral complex; MPFL, medial patellofemoral ligament; MQTFL, medial quadriceps-tendon femoral ligament.

patellar translation compared with the intact state in early degrees of flexion ( $0-45$ degrees). In addition, all three reconstruction states led to decreased lateral patellar translation compared with the sectioned state, with our results demonstrating statistical significance consistently from 0 through 30 degrees of flexion. Furthermore, although this study was not adequately powered to detect significant differences between reconstruction groups, we found a trend toward MPFL reconstruction providing the most robust patella stabilization with the smallest amount of lateral patella translation at 0 degrees of flexion, while MQTFL reconstruction demonstrated the largest translation.

Interestingly, we found that not only did the reconstruction groups provide less lateral patella translation compared with the sectioned state, they also provided significantly less patella translation when compared with the native intact state at early degrees of flexion (0-30 degrees). Given the fact that all of our grafts were fixated in full extension with the patella centered and no added tension applied, we believe this finding is likely related to the semitendinosus allografts providing more robust patellar stabilization than the anatomic MPFC. In fact, this claim is supported by prior publications investigating intrinsic tensile properties of the native MPFL compared with allograft tissue. LaPrade et al investigated the tensile strength of the intact MPFL and found that the mean load to failure was $178 \pm 46 \mathrm{~N}$ with a maximum of $270 \mathrm{~N}$ and a mean stiffness of $23 \pm 6 \mathrm{~N} / \mathrm{mm} .{ }^{17} \mathrm{~A}$ similar study by Mountney et al investigated 10 knees and found that the MPFL failed at a mean tension of $208 \pm 90 \mathrm{~N}$, with $70 \%$ of specimens failing due to a mid-substance tear. ${ }^{21}$ In comparison, a semitendinosus hamstring allograft can withstand 10 to 20 times this load to failure with one and two stranded grafts, respectively. The thickness of the graft also correlates with strength. In the present study, we used grafts 6 to $8 \mathrm{~mm}$ in diameter, which have been shown to withstand loads between $2,359 \pm 474$ and 3,908 $\pm 556 \mathrm{~N}$, respectively. ${ }^{19}$ Semitendinosus allografts also have increased stiffness when compared with native MPFL tissue. An investigation by Hamner et al found that single- and double-stranded semitendinosus grafts have a stiffness of $213 \pm 44$ and $469 \pm 185$ $\mathrm{N} / \mathrm{mm}$, respectively, which is $\sim 10$-fold stiffer than native tissue according to prior studies. ${ }^{17,20}$ These studies support that semitendinosus allografts are both intrinsically stronger and stiffer than the native MPFL that likely accounts for our finding of decreased lateral patellar displacement in the MPFC reconstructed knees when compared with the native knees.

Our results also demonstrate a trend between reconstruction groups at lower degrees of knee flexion. Although underpowered, MPFL reconstructions appeared to have the smallest amount of patella translation, while MQTFL reconstructions demonstrated the largest and hybrid reconstructions were in between the two. Further, when compared with the native state, the MPFL reconstruction group had the largest difference in patella translation, while the MQTFL demonstrated the smallest. This suggests that there may be a relationship between either the amount of patellar fixation, the number of graft limbs, and/or a combination of both, as it 
relates to patella stability and thus the amount of lateral patellar displacement. Although more robust fixation is seen in the MPFL reconstruction technique, with a higher number of graft limbs and more osseous patella fixation, there may be a tradeoff between the stiffness and robustness of the graft and subsequent complications. One of the more feared complications of MPFL reconstruction is patella fracture, which has been directly correlated to the type and amount of osseous patella fixation. ${ }^{10,21}$ The highest risk has been seen with transverse patellar bone tunnels with suggestions that the use of aperture fixation, such as suture anchor devices, can decrease the risk of patellar fracture. ${ }^{21-23}$ Some have even suggested the use of all-soft suture anchor patella fixation with a smaller bony footprint to further minimize the risk of patella fracture or iatrogenic articular penetration. ${ }^{24}$ Minimizing or even avoiding patella fixation altogether can negate the theoretic risk of patella implant related complications and is thus a major benefit of MQTFL and hybrid reconstruction techniques. With less osseous fixation, however, there is risk of less robust prevention of lateral patellar translation. We found that all three reconstruction techniques provided more stout patella stabilization at lower knee flexion angles than both the sectioned states and even the intact, native states. Interestingly, although MQTFL reconstruction uses only one graft limb without osseous patella fixation resulting in the least robust patella stabilization, it demonstrated the smallest difference in lateral patella translation when compared with the intact native state. Despite these findings in a cadaver model, it is unclear whether differences among these reconstruction groups would translate into any clinically significant differences. Therefore, it is important to understand the advantages and disadvantages between each reconstruction technique. Further, clinical studies are warranted to determine the optimal patella stabilization technique.

Conclusive evidence within the literature for correct graft tensioning during MPFC reconstruction remains limited. Beck et al reported that $2 \mathrm{~N}$ of graft tension during fixation adequately restores anatomic patellofemoral contact pressures; however, the appropriate knee flexion angle for graft tensioning remains highly debated within the literature. ${ }^{25-27}$ Appropriate placement of the graft within the native MPFL femoral insertion point as well as graft tensioning can have a significant effect on resultant lateral patella translation and overall knee kinematics. We dissected the anatomic insertion of the native MPFL to ensure appropriate femoral placement of our grafts and confirmed this placement by ensuring isometry of graft limbs throughout knee range of motion from 0 to 90 degrees of flexion. We performed final fixation of all grafts using minimal to no tension by a single surgeon (A.B.Y.) with the knee in full extension and the patella centered. Despite multiple published techniques recommending fixation of the graft in mild degrees of flexion, no clear evidence suggests a difference in outcomes with fixation in different knee flexion angles. Our results demonstrate significant differences in patella stability between the sectioned versus intact and reconstructed states only at early knee range of motion reinforcing the importance of the medial soft tissue stabilizers at lower knee flexion angles. Thus, appropriate length and tensioning of the graft may be best performed intraoperatively at lower knee flexion angles where the graft plays a more critical stabilizing role.

\section{Limitations}

There are several limitations that should be considered when interpreting the results of this study. First, our main limitation includes the use of cadaveric knees with an average age of $58.3 \pm 4.63$ years, which is greater than that of the typical patellar instability patient. Additionally, we did not evaluate cadaveric specimens for pathoanatomic risk factors that predispose to patellar instability including trochlear dysplasia, patella alta, elevated tibial tuberosity-trochlear groove distance, and femoral coronal or torsional malalignment. Although the presence of any of these variables could affect our results, testing every specimen in each state should still allow for adequate detection of changes in lateral patella translation between each state.

In this study, although we used vector forces totaling $175 \mathrm{~N}$ distributed through the quadriceps components as previously described, this amount and proportions are still theorized and have not been compared directly to in vivo loading. ${ }^{13}$ Further, cadaveric studies only allow static loading during testing that is in contrast to the dynamic nature of the stabilizing musculature in vivo. Furthermore, quadriceps loading may have a disproportionate effect on patella stability in MQTFL and hybrid reconstruction states when compared with MPFL reconstruction states as the VMO is more intimately involved in these constructs. Further, we noticed increased sample variance at each tested state that was likely secondary to slight variations in the angle of the loading vectors secondary to human error. It is possible that our results may have varied if there were significant changes in extensor mechanism vector loading.

A final limitation to consider is our sample size of six specimens, rendering this study not adequately powered to detect significant differences between each reconstruction group. Utilizing the means and standard deviations from our final data, a post hoc power analysis for an analysis of variance with an $\alpha$ of 0.05 would need at least 172 cadavers per group to be powered at $80 \%$. This is obviously not feasible with a cadaveric study and thus our main focus was to detect significant changes in patellar displacement between reconstruction groups and the intact and sectioned groups. With this approach, a power analysis supported the need for four specimens between groups to detect a clinically significant difference in displacement, defined as $4 \mathrm{~mm}$ of lateral patella translation. Despite this, our smaller sample size likely explains why some findings we expected to be significant, such as the difference between intact and sectioned states at full extension, only trended toward significance. Additionally, the small decrease in patella translation at high knee flexion angles (60 and 90 degrees) seen in the sectioned state compared with the intact state again is likely due to normal variation in a small sample set and thus clinically insignificant. Despite these aforementioned limitations, our study represents a novel investigation of three MPFC reconstruction techniques with valid and significant findings. 


\section{Conclusion}

This biomechanical cadaveric study demonstrated the efficacy of three different MPFC reconstruction techniques in restoring patella stability following MPFC sectioning, especially at lower knee flexion angles where the medial soft tissue restraints play a more important role. Future clinical studies are needed to investigate the clinical correlates of these findings.

\section{Authors' Contributions}

W.M.C. and H.P.H. substantially contributed to study conception and design, biomechanical testing, analysis, and interpretation of the data, drafting and revising, approved the final version, and agrees to be accountable for all aspects of the work. E.F.S. substantially contributed to study design, biomechanical testing, drafting and revising, approved the final version, and agrees to be accountable for all aspects of the work. B.J.C. and A.B.Y. were involved in substantial contributions to study conception and design, interpretation of the data, drafting and revising, approved the final version, and agrees to be accountable for all aspects of the work. All authors contributed substantially to conducting the underlying research and drafting of the manuscript. All authors reviewed and approved the final manuscript.

\section{Conflict of Interest}

None declared.

\section{References}

1 Fithian DC, Paxton EW, Stone ML, et al. Epidemiology and natural history of acute patellar dislocation. Am J Sports Med 2004;32 (05):1114-1121

2 Sanders TL, Pareek A, Hewett TE, Stuart MJ, Dahm DL, Krych AJ. Incidence of first-time lateral patellar dislocation: a 21-year population-based study. Sports Health 2018;10(02):146-151

3 Tanaka MJ, Chahla J, Farr J II, et al. Recognition of evolving medial patellofemoral anatomy provides insight for reconstruction. Knee Surg Sports Traumatol Arthrosc 2019;27(08):2537-2550

4 Desio SM, Burks RT, Bachus KN. Soft tissue restraints to lateral patellar translation in the human knee. Am J Sports Med 1998;26 (01):59-65

5 Aframian A, Smith TO, Tennent TD, Cobb JP, Hing CB. Origin and insertion of the medial patellofemoral ligament: a systematic review of anatomy. Knee Surg Sports Traumatol Arthrosc 2017;25 (12):3755-3772

6 Hautamaa PV, Fithian DC, Kaufman KR, Daniel DM, Pohlmeyer AM. Medial soft tissue restraints in lateral patellar instability and repair. Clin Orthop Relat Res 1998;(349):174-182

7 Conlan T, Garth WP Jr, Lemons JE. Evaluation of the medial softtissue restraints of the extensor mechanism of the knee. J Bone Joint Surg Am 1993;75(05):682-693

8 Feller JA, Richmond AK, Wasiak J. Medial patellofemoral ligament reconstruction as an isolated or combined procedure for recurrent patellar instability. Knee Surg Sports Traumatol Arthrosc 2014;22(10):2470-2476

9 Stupay KL, Swart E, Shubin Stein BE. Widespread implementation of medial patellofemoral ligament reconstruction for recurrent patellar instability maintains functional outcomes at midterm to long-term follow-up while decreasing complication rates: a systematic review. Arthroscopy 2015;31(07):1372-1380

10 Dhinsa BS, Bhamra JS, James C, Dunnet W, Zahn H. Patella fracture after medial patellofemoral ligament reconstruction using suture anchors. Knee 2013;20(06):605-608

11 Uppstrom TJ, Price M, Black S, Gausden E, Haskel J, Green DW. Medial patellofemoral ligament (MPFL) reconstruction technique using an epiphyseal femoral socket with fluoroscopic guidance helps avoid physeal injury in skeletally immature patients. Knee Surg Sports Traumatol Arthrosc 2019;27(11):3536-3542

12 Fulkerson JP, Edgar C. Medial quadriceps tendon-femoral ligament: surgical anatomy and reconstruction technique to prevent patella instability. Arthrosc Tech 2013;2(02):e125-e128

13 Christian DR, Redondo ML, Cancienne JM, et al. Differential contributions of the quadriceps and patellar attachments of the proximal medial patellar restraints to resisting lateral patellar translation. Arthroscopy 2020;36(06):1670-1676

14 Cancienne JM, Christian DR, Redondo ML, et al. The biomechanical effects of limited lateral retinacular and capsular release on lateral patellar translation at various flexion angles in cadaveric specimens. Arthrosc Sports Med Rehabil 2019;1(02):e137-e144

15 Amis AA, Firer P, Mountney J, Senavongse W, Thomas NP. Anatomy and biomechanics of the medial patellofemoral ligament. Knee 2003;10(03):215-220

16 Bicos J, Fulkerson JP, Amis A. Current concepts review: the medial patellofemoral ligament. Am J Sports Med 2007;35(03):484-492

17 LaPrade MD, Kallenbach SL, Aman ZS, et al. Biomechanical evaluation of the medial stabilizers of the patella. Am J Sports Med 2018;46(07):1575-1582

18 Mountney J, Senavongse W, Amis AA, Thomas NP. Tensile strength of the medial patellofemoral ligament before and after repair or reconstruction. J Bone Joint Surg Br 2005;87(01):36-40

19 Boniello MR, Schwingler PM, Bonner JM, Robinson SP, Cotter A, Bonner KF. Impact of hamstring graft diameter on tendon strength: a biomechanical study. Arthroscopy 2015;31(06):1084-1090

20 Hamner DL, Brown CH Jr, Steiner ME, Hecker AT, Hayes WC. Hamstring tendon grafts for reconstruction of the anterior cruciate ligament: biomechanical evaluation of the use of multiple strands and tensioning techniques. J Bone Joint Surg Am 1999;81(04): 549-557

21 Parikh SN, Wall EJ. Patellar fracture after medial patellofemoral ligament surgery: a report of five cases. J Bone Joint Surg Am 2011;93(17):e97-1-8

22 Parikh SN, Nathan ST, Wall EJ, Eismann EA. Complications of medial patellofemoral ligament reconstruction in young patients. Am J Sports Med 2013;41(05):1030-1038

23 Shah JN, Howard JS, Flanigan DC, Brophy RH, Carey JL, Lattermann C. A systematic review of complications and failures associated with medial patellofemoral ligament reconstruction for recurrent patellar dislocation. Am J Sports Med 2012;40(08):1916-1923

24 Saper MG, Meijer K, Winnier S, Popovich J Jr, Andrews JR, Roth C. Biomechanical evaluation of classic solid and all-soft suture anchors for medial patellofemoral ligament reconstruction. Am J Sports Med 2017;45(07):1622-1626

25 Beck P, Brown NAT, Greis PE, Burks RT. Patellofemoral contact pressures and lateral patellar translation after medial patellofemoral ligament reconstruction. Am J Sports Med 2007;35(09): 1557-1563

26 Kita K, Horibe S, Toritsuka Y, et al. Effects of medial patellofemoral ligament reconstruction on patellar tracking. Knee Surg Sports Traumatol Arthrosc 2012;20(05):829-837

27 Lorbach O, Zumbansen N, Kieb M, et al. Medial patellofemoral ligament reconstruction: impact of knee flexion angle during graft fixation on dynamic patellofemoral contact pressure-a biomechanical study. Arthroscopy 2018;34(04):1072-1082 